\title{
ML Based Smart Energy Meter Observing \& Bill Supervision Using Raspberry Pi
}

\author{
V. Amala Rani ${ }^{1, *}$, T. Thaj Mary Delsy ${ }^{1}$, D. Marshiana ${ }^{1}$, N. Praveen ${ }^{1}$, P. Kartheek ${ }^{1}$ \\ ${ }^{I}$ Department of Electronics and Instrumentation Engineering, Sathyabama Institute of Science and Technology, \\ Chennai,600119, Tamilnadu, India \\ ${ }^{*}$ Corresponding author. Email: v.amalarani@gmail.com
}

\begin{abstract}
Nowadays the power consumption is increasing unknowingly in our houses and Some faulty devices will consume more energy than what it is taken. So, to overcome those defects a system is being proposed that will provide the information about the power consumption and faulty loads by the system and also, to know the power consumption and billing amount in the cloud in graphical representation. This system can reduce the time and also make the entire process into a smart one. By using this system manual calculations will be avoided. A novel process was developed based on Raspberry pi microcontroller to identify and monitor the power consumption, faults, bill supervision. A data will be measured automatically and passed to the cloud server protocol through IOT module. The data will be sent to cloud by the help of current transformer and potential transformer. The terms like power consumption, bill supervision in cloud (ThingSpeak) can be seen anywhere in the country. The system will keep track of power consumption and bill supervision. Then whole data will be updated to cloud (ThingSpeak) for instant of time. The data will be updated to cloud for every 180 seconds. Internet is necessary for the system to update collected data to cloud (ThingSpeak).
\end{abstract}

Keywords: Raspberry pi, IOT, power consumption, Things Speak.

\section{INTRODUCTION}

Electricity is one of the vital requirements for sustainment of contents of life. It should be used very judiciously for its proper utilization. Electronic metering technology vastly reduces the man power and time [1]. It also avoids the mistakes due to manual calculation. Meters used in day-to-day life uses normal electromechanical meters with reduced accuracy. So, the conventional electromechanical meters are being replaced by new electronic meters to get better precision in measuring the data from meter. Here the load which is connected to the system will be monitored and examined through online using Things-peak cloud analysis. The user at first needs to create account in Things-peak. Things-peak unique channel id is required to analyse power consumption and bill super vision. By using this system, how much power consumed is known and also, the billing amount is generated based on consumed power.

\section{BACKGROUND}

The newer system repeatedly measures the energy meter information and stores it in cloud. In the existing system, by using GSM technology, a wireless automatic reading system is implemented with Raspberry Pi microcontroller [2]. The energy meter reading is also implemented using IOT and Rasperry Pi [3] by Satish Kumar Pal et.al. Current monitoring a using IOT and smart speaking Energy meter is implemented by L Aro Franklin et.al and also for current theft detection using Raspberry Pi [4]. Using ardino, a GSM based energy meter is implemented by Brinda et al. [5]. The meters used for energy measurement has the capability of reading only $\mathrm{kWh}$ units. These units are measured by human meter reader or IR sensors [6] for every month. The reading information can be delivered to the customers through SMS services [7]. The measured data are to be handled and maintained by a authority for reference by IOT [8] or with respect to Wi-Fi [9]. Normal electric meters, provides power consumption [10], readings .to remote places for communication. It is a sole communication method for interface. It only measures instantaneous values and validation using the Enermon [11] for low range system monitoring [12]. The drawback of existing system is as follows. Highly depends on meter reading. Human errors $[13,14]$ may occur for the manual meter reading. More number of employees needed and extra Expensive to the company for hiring them. The 
consumer will not receive any information of his expected usage of power.

\section{METHODOLOGY}

In this system a smart energy meter was developed and implemented in the houses or factories for the communication purpose with energy meter to the electricity board. The communication is based on wireless data protocol, which can operate along with the IOT module. This system consists of a Raspberry pi(microcontroller) along with the digital energy meter for measurement. The current unit measurement from energy meter is transferred to cloud portal with the help of transformers (current and potential) by IOT module. The stored data can be made on familiar with customer name and various parameters like power loss, over power usage, instantaneous power, total energy usage, faulty loads, can be recognized by this system. Every branch in the power system can be monitored in every instant of time. There are two branches of loads are there in the block diagram (fig.1). For those branches' separate voltage and current transformer pairs are added each will continuously monitor the instantaneous power. The Raspberry pi software is used as a communication device and also to store the information regarding the meter reading. Whenever loss occurred the value of dataset will be compared with present values and the fault will be determined, fault in a particular branch can also be determined so that it will be easy for regulating those faults. If any fault occurred it can also be determined by regular check-up, also faulty load can be fined by this system. In the proposed model the main advantage of the system is connected to ML which helps in big data analysis so the queue formation of data can be avoided. The model used in now a days require manual reading and it will be uploaded manually so the data can have human error. It is overcome by the proposed model due to the analysis and pre-processing of data, the error occurrence can be neglected and automatic storage of information to the electricity board.

\subsection{System Hardware}

The hardware required for this system is as follows. Figure 1 represents the operational block of the proposed system. In the system it determines the operation carried out in billing process. The software module and hardware module are interfaced and connected to the cloud portal. In Figure 2 represents the data transfer through the cloud portal which is based on ML based thinkspeak analysis. The development machine learning based on the back propagation algorithm was implemented in smart energy meter. The software program was developed to measure the reading automatically without the need of human interference and to provide automatic billing. The low cost single board computer type Raspberry $\mathrm{Pi}$ is used for programming. The modified version of Debian Linux was used for the control purpose and it was optimized for the ARM architecture, $3^{\text {rd }}$ Gen, $700 \mathrm{MHz}$ ARM Applications Processor. with 1 GB RAM.

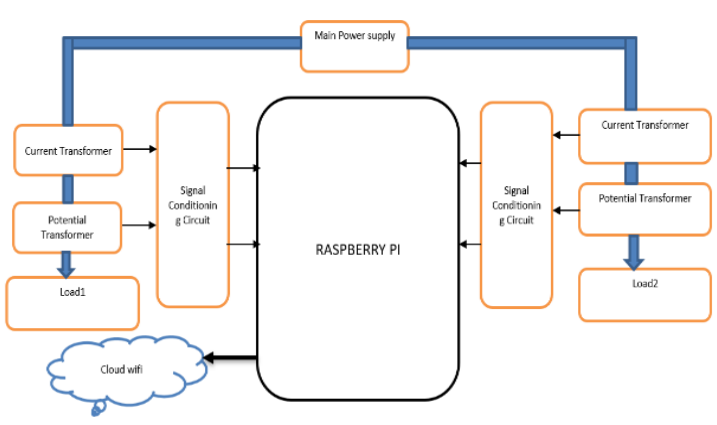

Figure 1 Block diagram of proposed system

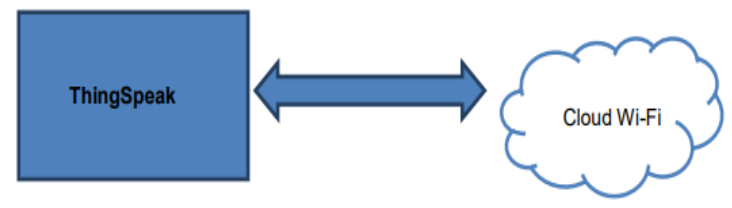

Figure 2 Cloud Analysis

The figure 3 represents the frame work for machine learning system used in energy meter. The machine learning technique includes the neural network algorithm.

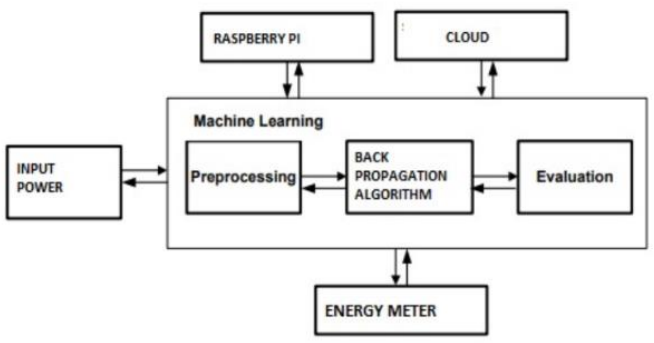

Figure 3 Frame work of Machine Learning

In ML the preprocessing of input data from the power consumed is accountable by the input data. The algorithm used is a back propagation it is used to determine the error value to provide the information for overloading. The evaluation of data is to be processed so the values can be evaluated by using the training technique. It includes the relation with the raspberry pi for the analysed data from the ML to be related for the wireless transmission. The thinkspeak analysis is connected through ML for data transmission using cloud portal. The current transformer is connected to raspberry pi. The current transformer is used for measure the current level for the given load. Current transformers decrease high voltage currents to much lower and supplies the convenient way of safely monitoring the actual electrical current. 


\subsection{Potential transformer}

The voltage transformer is connected to raspberry pi. The voltage transformer is used for measure the voltage level for the given load. The co-axial winding is used in the potential transformer so as to minimize the leakage reactance.

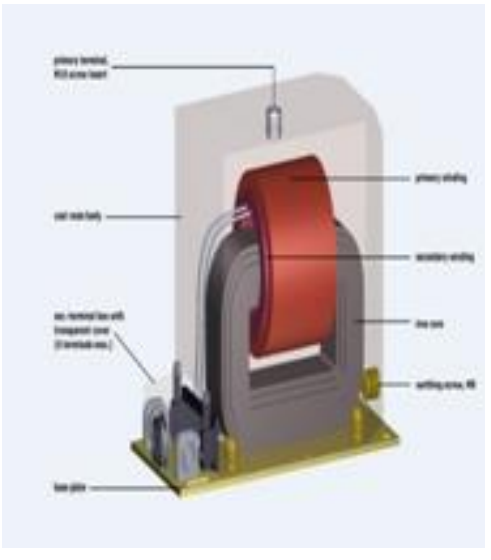

Figure 4 Potential transformer

Figure 4 is the hardware unit of Energy meter is used for observing and bill supervision. The method was ML based on Raspberry pi implemented in microcontroller to detect and monitor the power consumption, faults, bill supervision. This can reduce the time and also make the entire process into a smart one. The data will be updated to cloud for every 180 seconds. Internet is necessary for the system to update collected data to cloud (ThingSpeak). Figure 5 represents the hardware module.

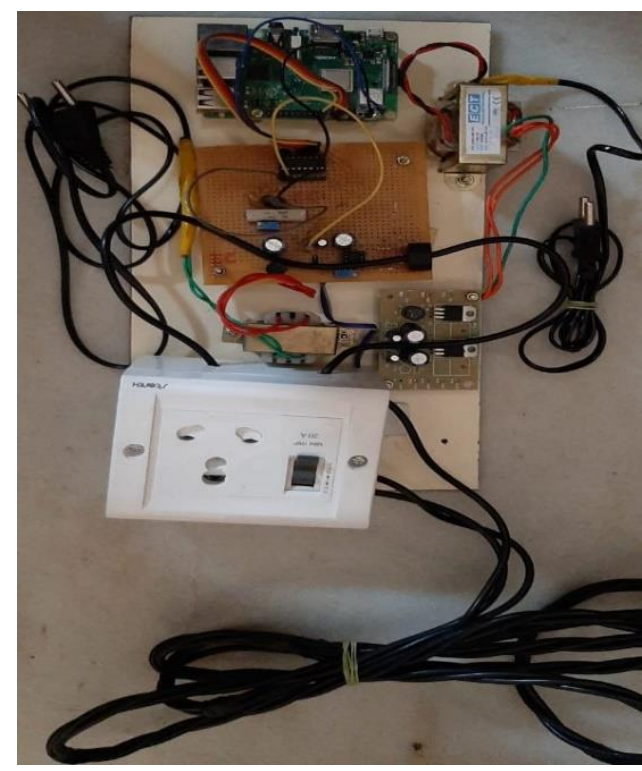

Figure 5 Hardware module

\section{RESULTS AND DISCUSSIONS}

This smart energy meter was implemented by using Machine learning. The newly developed system measures the energy meter data without human intervention and stores it in Thingspeak cloud. This system tests for a load in three states, i.e. (i) No load (ii) Normal load (iii) Risk load. The data (Power consumption and bill supervision) for a given load will be automatically sent to cloud account. a fourth level heading. You can replicate it where suitable.

\subsection{No load}

The current investigation represents no load condition. Here no load should be connected to the system. Figure 6 represents the current status of the given load.

The figure 6 shows no load condition in graphical representation in Thingspeak (cloud). It shows power consumption by given load (Field 2 Chart) and bill supervision of given load in Field 1 Chart.

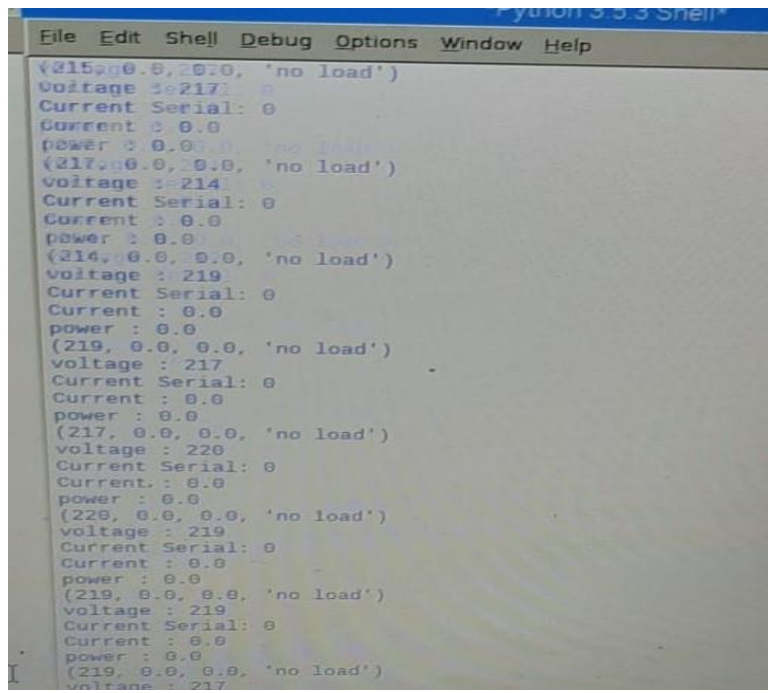

Figure 6 No load

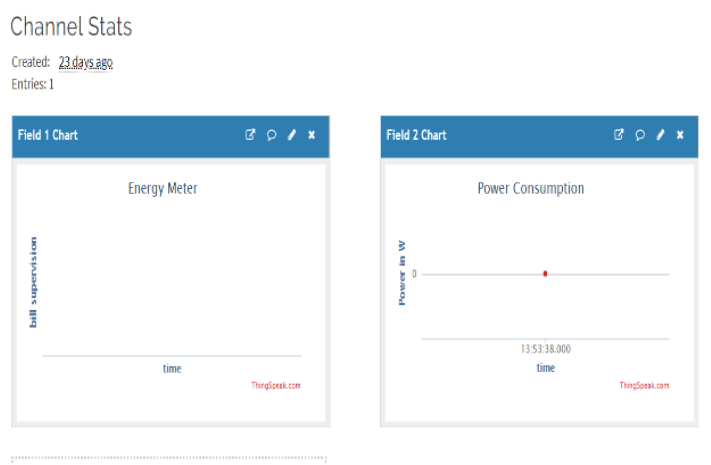

Figure 7 Thingspeak view for no load

\subsection{Normal load}

The current investigation represents normal load condition. A load should be connected to the system. The figure 8 represents the current status of the given load. 


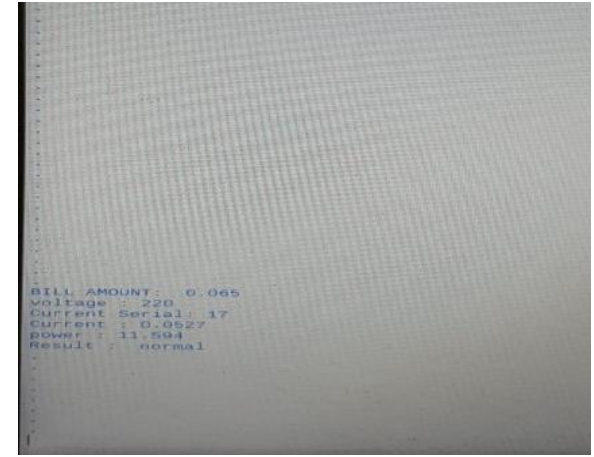

Figure 8 Normal load

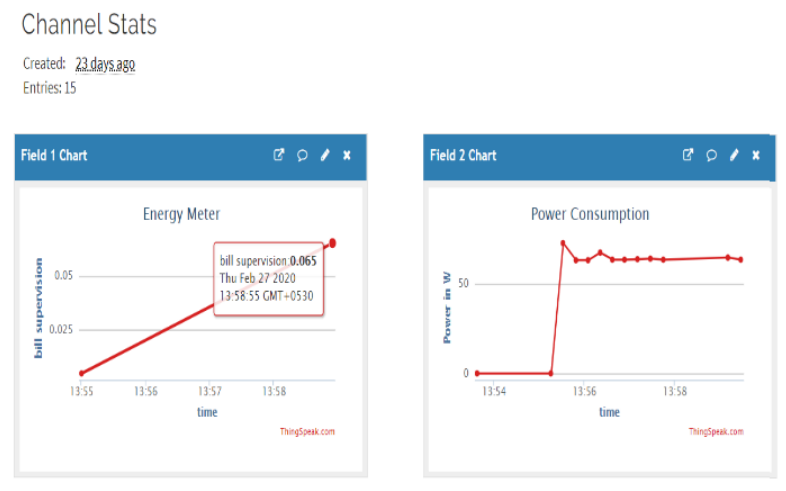

Figure 9 Thingspeak view for normal load

The figure 9 shows normal load condition in graphical representation in Thingspeak (cloud). It shows power consumption by given load (Field 2 Chart) and bill supervision of given load in Field 1 Chart.

\subsection{Risk load}

The current investigation represents risk load condition. Here a fault load (risk load) should be connected to the system. Figure represents the current status of the given load.

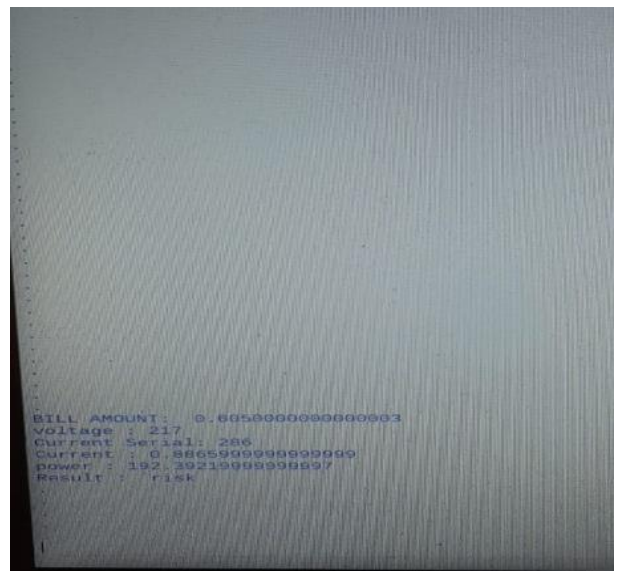

Figure 10 Risk load

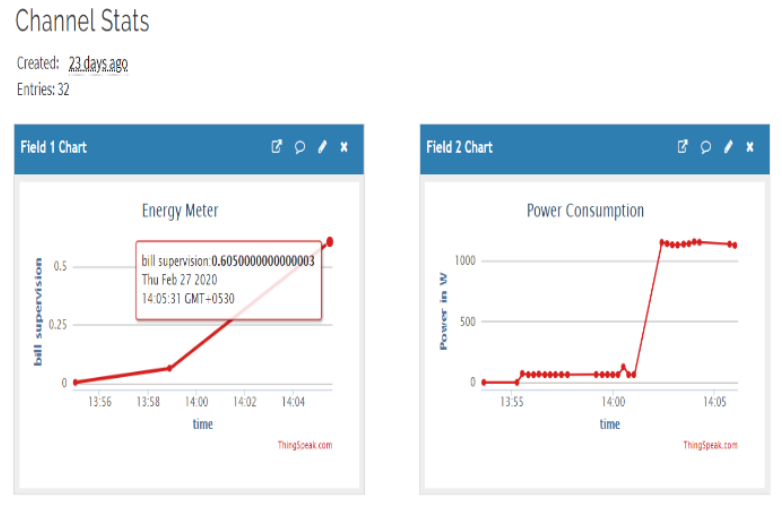

Figure 11 Thingspeak view for risk load

The figure 11 shows risk load condition in graphical representation in Thingspeak (cloud). It shows power consumption by given load (Field 2 Chart) and bil supervision of given load in Field 1 Chart.

Table 1. Load condition

\begin{tabular}{|l|l|l|}
\hline Load condition & $\begin{array}{l}\text { Load } \\
\text { value(Power) }\end{array}$ & Information \\
\hline No Load & 0 & - \\
\hline Normal Load & 11 & - \\
\hline Risk Load & 192 & Risk \\
\hline
\end{tabular}

\section{CONCLUSIONS}

The newer development of energy meter and bill supervision can able to control the amount of power consumed by the customer. The faulty devices are identified and the wastage of power can be avoided. This entire data i.e.; power consumption and bill supervision will be sent to cloud (ThingSpeak). The entire output could be seen in cloud in graphical representation. This method would be more beneficial for all real time applications. this method reduces the human effort and also parallax error due to the automatic reading collection from the meter. the calculation of payment details will be updated to the customer so excess amount payment can be reduced. This system is more consistent, precise and user friendly. The result will be monitored in cloud anywhere in the world.

\section{REFERENCES}

[1] Md. Mejbaul Haque, Md. Kamal Hossai, Md. Mortuza Ali, Md. Rafiqul Islam Sheikh, "Microcontroller Based Single Phase Digital Prepaid Energy Meter for Improved Metering and Billing System", International Journal of Power Electronics and Drive System, Vol.1, No.2, December 2011, pp. 139 147

[2] Sneha Salunkhe, Dr. S. S. Lokhande, "Raspberry Pi based automatic meter reading", International 
Journal of Application or Innovation in Engineering \& Management, Volume 5, Issue 9, September 2016 ,pp.69-73.

[3] Satish Kumar Pal, Amit Ranjan, Abhishek Mishra , Chandra Prakash Singh, Amitabh Srivastava, "Electric meter reading through IOT by using Raspberry pi model B2", International Research Journal of Engineering and Technology, Volume: 05, Issue: 03 , Mar-2018 ,pp-3603-3608.

[4] L Aro Franklin, R Dharun, D ManiMaran, A Jaya Prassath, P Bency, "IoT Based Monitoring and Controlling of Smart Speaking Energy Meter and Current Theft Detection using Raspberry pi 3", International Research Journal of Engineering and Technology, Volume: 06, Issue: 03, Mar 2019 ,pp1975-1978.

[5] Brinda.S , Vishal Kumar Sah , Jaladi Harish , Akshay.U, Vishal Deo Mahto, Swetha Umapathy, "Smart Energy Meter "International Journal of Engineering Science and Computing, vol.8,no.3, 2018,pp 16578-16581.

[6] Moni silviya .E, Meena vinodhini .K, "GSM based automatic energy meter with instant building",International Journal of advanced research in Electrical, Electronics and Instrumention Engineering, vol.no-03, April 2014, page.no 44-49.

[7] Prof. kurku.S.R, “Automatic Energy Meter Reading System Using GSM Technology”. International journal of innovative research in electrical electronics, instrumentation and control engineering, Vol.no:04, year:March 2016, pp. 149152.

[8] Narmadha, R., Immanuel Rajkumar, R. Sumithra, and R. Steffi. "Continuous Monitoring of Electricity Energy Meter Using IoT." In Soft Computing for Problem Solving,, pp. 731-738. Springer, Singapore, 2020.

[9]J.AdelineSneha,V.AmalaRani,T.V.SubhaAustaleksh mi,J.Aran Glenn,“ Comparison of Boiler Control Using Wireless Bridge Between PLC, SCADA, WiFi., International Journal of Applied Engineering Research,10(1),2015,49-57.

[10] Yoeseph, N \& Safi'ie, M \& Purnomo, F. Smart Energy Meter based on Arduino and Internet of Things. IOP Conference Series: Materials Science and Engineering. ,2019.

[11] Diogo Santos, João C. Ferreira 1, "IoT Power Monitoring System for Smart Environments",Sustainability, 2019, 11, 5355

[12] Prof.Ganesh K. Shirsat, Nilesh U. Bhangale, Urvesh Y. Gurav, Suraj A. Jawale, "IoT Based Energy
Monitoring System for Energy Conservation", International Research Journal of Engineering and Technology, vol.7,4,April 2020, pp 758-763.

[13] A. Sundaram 1 ,Dr. G.P. Ramesh "Sensor less Control of BLDC Motor using Fuzzy logic controller for Solar power Generation" International Journal of MC Square Scientific Research, vol.9,No.2,2017, pp 70-79.

[14] Ramesh, G. P., and K. S. Gowrishankar. "Enhancement of power quality and energy storage using a three-terminal ultra capacitor and CCM converter for regenerative controlled electric drives." Int J Emerg Res Manag Technol, vol.9,No.2,2012,pp. 56-61. 